\title{
Smart Basket : The Modern era IoT Application
}

\author{
Talreja Sahil ${ }^{1}$, Pendharkar Arjun ${ }^{1}$, Madur Srushti ${ }^{1}$, Mohammad Saad Nalband ${ }^{1}$, Pragati Mahale ${ }^{2}$ \\ ${ }^{1}$ Information technology, B.E AISSMS's Institute of Information Technology, Pune, Maharashtra, India \\ ${ }^{2}$ Information Technology, Assistant Professor, AISSMS's Institute of Information Technology, Pune, Maharashtra, India
}

\begin{abstract}
Article Info

Volume 8, Issue 2

Page Number : 585-593

\section{Publication Issue}

March-April-2021

\section{Article History}

Accepted : 15 April 2021

Published : 25 April 2021

Smart basket is a technology that uses Arduino and RFID. During pandemic times like Covid-19 there gets the crowd in the grocery marts, and the customer must wait in queue for final billing. But there may be high risk during these times of spreading the disease, so Smart basket brings an ideal solution for getting the final billing done. Using Arduino and RFID tag customer gets a final bill through the web app which gives a list of products scanned by the smart basket. The customer can remove the products which he further wishes to eliminate from his basket and delete the item from the final bill in the web app which can result in savings too. This smart basket also reduces manpower as the traditional method of buying the products involves a long time, with RFID tag the same thing is performed in a modern way.
\end{abstract}

Keywords : Arduino, RFID tag, RFID reader, Shopping cart, Wireless Communication.

\section{INTRODUCTION}

Smart basket is a technology used inside small grocery shops to big malls. With the use of an RFID tag and RFID reader, shopping is made easy. During times like pandemic or festive, there is a lot of crowds in malls. Overcrowding is the biggest issue in continents like Asia and Africa. But with evolving, IoT technology new solution devices are made each month. With Arduino, the technologies had a huge impact on human lives. With the use of Arduino and RFID technology, we had made a Smart basket that will use a Wi-Fi module. The customer must scan a product on an RFID reader, the products in near future will come with In-built RFID tag chips on the product. The product details will be read by the RFID reader and a web application will be there where these scanned product details will be present. The customer must pay the final bill in any mode of payment and generate E-receipt. This way the Smart basket is avoiding overcrowding in the malls while customers must scan a product on the go. In past this technology had evolved a lot and yet must bring some new changes. We had discussed and used RFID technology and Node MCU(ESP2866). In this following paper, we had presented a survey on different techniques used in Smart Basket over the years. 
It brings the ideal solution in this modern era, IoT appliances are evolving so is technology, which results in reduced manpower. Gone are the days where the customer must wait in long queues for getting the final billing done for his products and wait to exit with requiring tedious efforts. Arduino platform and RFID technology are bringing a solution embedded in the smart basket which helps the customer to get the best experience in shopping.

People are worried about how they can buy their favorite products during times like pandemic and a lot of restrictions which makes the sales of mall decrease and people face problems. But now IoT applications have made human life easy so people can save their time and get satisfaction.

With technology there exists pros and cons, when purchasing products there are lots of items from grocery essentials to electronic appliances, the customer must place the items and then go to checkout. The counter cashier must scan the barcode and make the final bill but IoT and Arduino make the process in a basket which saves times, with this shopper saves times and the bill is generated automatically with saving paper for environment. There is continuous development in domestic industry with these types of similar technology to enhance customer experience and save time and help the world. This strategy can help in smart scaling, wireless automation, and more scope in IoT embedded software and hardware.

Thus, this paper highlights our concern on a Smart basket that is solely based on IoT technology, which is an area evolving with changing world. In the use of this system, the shoppers will scan each item with the RFID reader, then the information about the products is displayed on a web application with the total amount due pending and when added payment method, the payment can be done automatically and immediately. [2]The microcontroller is placed beneath the basket and the RFID tag reader and scanner is embedded alongside the Arduino hardware to scan and send the details of the product and the web app makes customer invoice.

Section 2 explores the literature review of the following paper. Section 3 demonstrates the proposed model, Section 4 highlights Implementation, Section 5 glimpses on Methodology of the Smart Basket and in the last section we had discussed the conclusion and future scope of Smart Basket using IoT technology.

\section{LITERATURE SURVEY}

\section{Smart Shopping Cart using RFID \& Zigbee.}

The smart basket technology had evolved a lot in the recent years, one such technique used was Zigbee device. This device is based on IEEE 802.15 standard. Zigbee is a device which transmit data covering longer distances. It passes data through intermediate devices to reach more longer ones i.e., devices, resulting in a mesh network. The RFID and Zigbee technology brought an automated billing system. The proposed system was as follows: Inside a shopping mall, each shopping cart was designed in such a way that each shopping basket would contain a RFID device i.e., to identify product. This was the product identification device which contained the microcontroller. As the customer would proceed to buy products the customer has to scan the product through RFID reader, the RFID reader reads the product details through the RFID chip. The basket containing product identification device also contains microcontroller, LCD, an RFID reader, EEPROM, and ZigBee module. The information read by the RFID reader is stored inside the EPROM and this data is sent to billing system by Zigbee module.

RFID is the radio frequency identification which is the on-going technology and progressing each day. It has been helping the IoT industry grow more and more towards sustaining environment and automation. The technology is old but with latest 
trends in technology world RFID chips are made for ease of use of the humans. This technology is bringing and will change the supply-chain industry and logistics.

Zigbee is a network device which is built on top the IEE 802.15.4 standard. Creating a mesh network and transmitting the data to distant device is done by Zigbee module. It provides wireless networking protocol at low cost with low data rate and power consumption which helps toward automation of remote-control applications.

\section{Smart Trolley using RFID.}

In this product the RFID technology was used with IR technology. The infra-red technology is another technology which was used in past years to get the Smart trolley work inside the mall. The system also contains ARM processor like in past microcontroller for processing of the desired data. The concept of smart shopping cart there was a use of IR sensor and ARM processor which made alert for the customer whether a product has been removed. This was introduced due to theft and fraud or any disputes between customer and shop owner., and smart shopping trolley but depending upon growing technology, need of the market and hardware cost each year the products must bring some changes. In this product.

This product used a RS232 protocol which helps in the processing for billing purpose along with ARM processor. The working was simple and like past products but with ease of use. The customer must scan the RFID tag i.e., a chip on RFID reader the details of the product will be sent to system memory. As the customer scans the product there will be a option to check whether the product is available or not on LCD display. If the product is found the basic details such as name and price on LCD display. The product details are processed in a way where ARM processor and the protocol i.e., RS232 will work to calculate and process the data. The IR sensor mentioned before is used for counting purpose i.e., to count the number of products. The sensor will emit the rays in continuous fashion and help in storing the products in ARM processor. After completion of the shopping, there is a button which when pressed indicates the final billing of the products on a webbased interface.

\section{PROPOSED WORK}

The goal of the system is to deliver a system that is easy to work and generate automated invoices. Making this system easily accessible and deliver the shopping system with low-cost hardware, which will bring an ideal solution both for consumers and retailers. The basket will contain the sensor module with a circuit such that when an item is placed it will be scanned with an RFID reader, the product should include an RFID tag. When the item is scanned, and RFID has completed its work, the microcontroller will store the data using SPI protocol with and Node MCU [4] will help this data to store in the system. In this way, the sensor module will have Arduino embedded in the circuit with all necessary hardware components.

When the customer enters the shopping mall, he/she will take the basket. [3] The basket has the sensor module which contains an RFID reader per customer taking that basket, with customer inspecting the products they must scan the product through RFID tag and the RFID tag will send this detail to the RFID reader, which relates to Arduino (Arduino contains inbuilt microcontroller) now the Node MCU Wi-Fi module (ESP2866) will communicate with the database using SPI protocol and the products will be stored on the webpage of the shopper's website. The scaling and automation help in regulating the process easy and the bill is generated fast. 


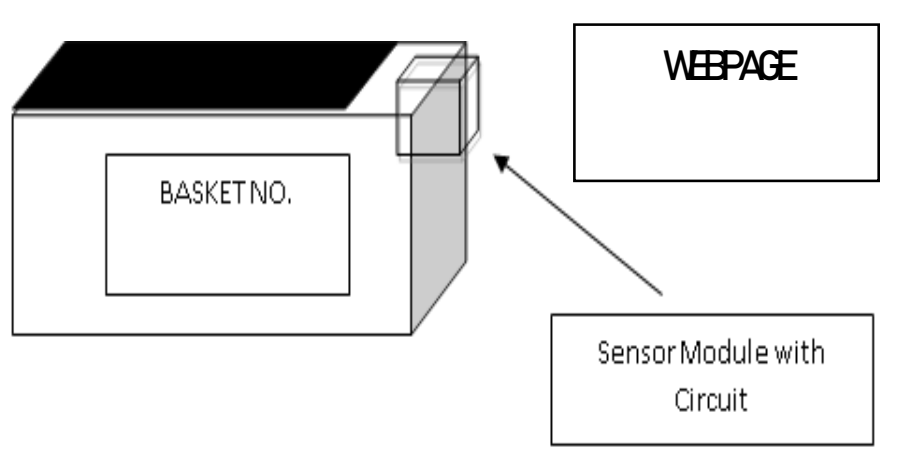

Figure 1: Design of setup.

\section{IMPLEMENTATION}

The product can be demonstrated as follows, and one thing noteworthy is it provides the flexibility to carry out as a portable device in the IoT appliances.

\section{Hardware System}

The following image has two RFID readers, one RFID tag, and Node MCU(ESP2866) module. These all three make up a sensor circuitry system to be placed below the smart basket. Once the customer scans the product barcode through an RFID tag, the data will be sent to an RFID reader and the Arduino code start implementing SPI protocol which helps the microcontroller to communicate with a web application by HTTP protocol on the Internet.

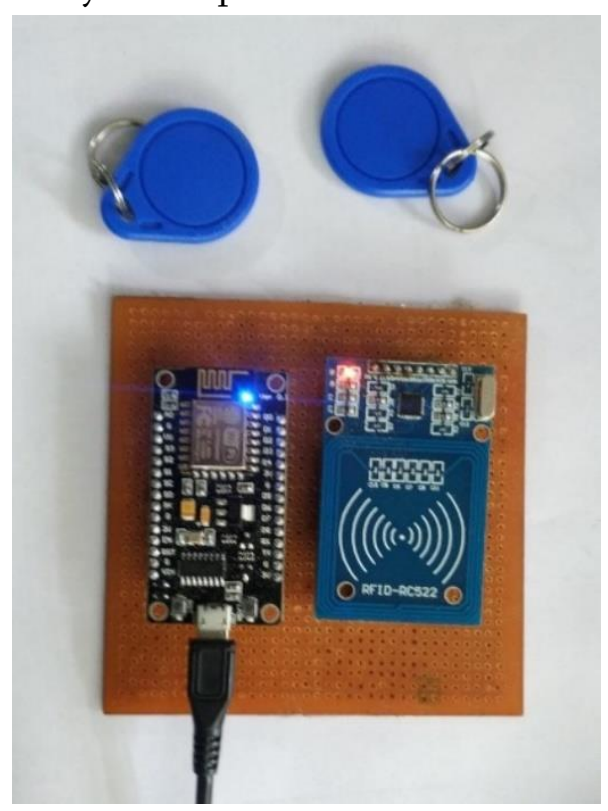

Figure 2: Smart Basket Circuitry system.
The reader receives the data from the tag which in turn makes the microcontroller compute the data. The Wi-Fi module will send this data to a web page which will be stored in the Database. The Database used is RDBMS (Relational Database Management System) which is using MySQL for storing this data. The checkout page has multiple modes of payment to offer such as Cash, Cheque, Card, Digital Wallet

\section{Software System}

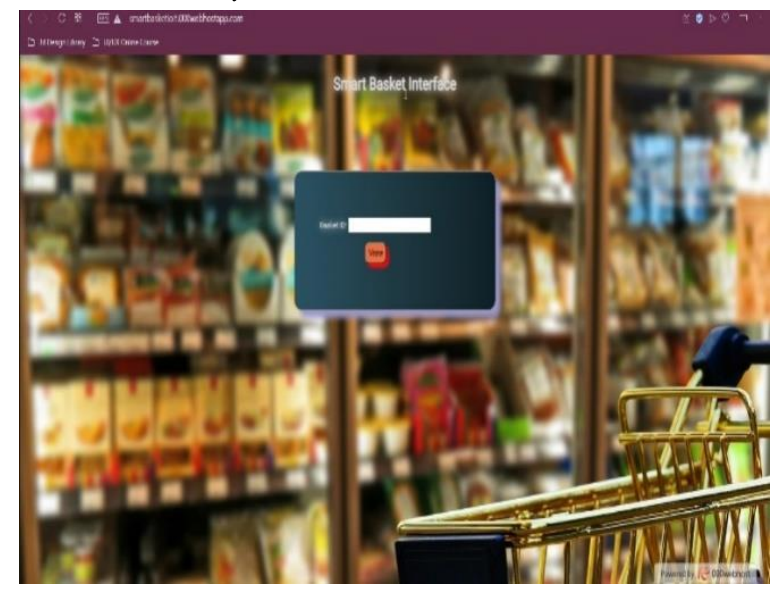

Figure 3. Login Page of Basket ID for the customer

The above image is the initial login page of the Smart Basket when the customer arrives in the store. The basket which he takes inside the mall is the basket ID for his whole shopping journey process inside the mall. He can check the list of products and their price on the check-in page. On the check-in page, there is a table that will help the customer to see the name of the product he scanned, the quantity and price of the product. This way he can ensure fast billing and getting invoices. Additionally, he can remove a particular product which he does not desire for.

Finally, he can choose various modes of cash payment which he wishes for and check out in the fastest mode. 


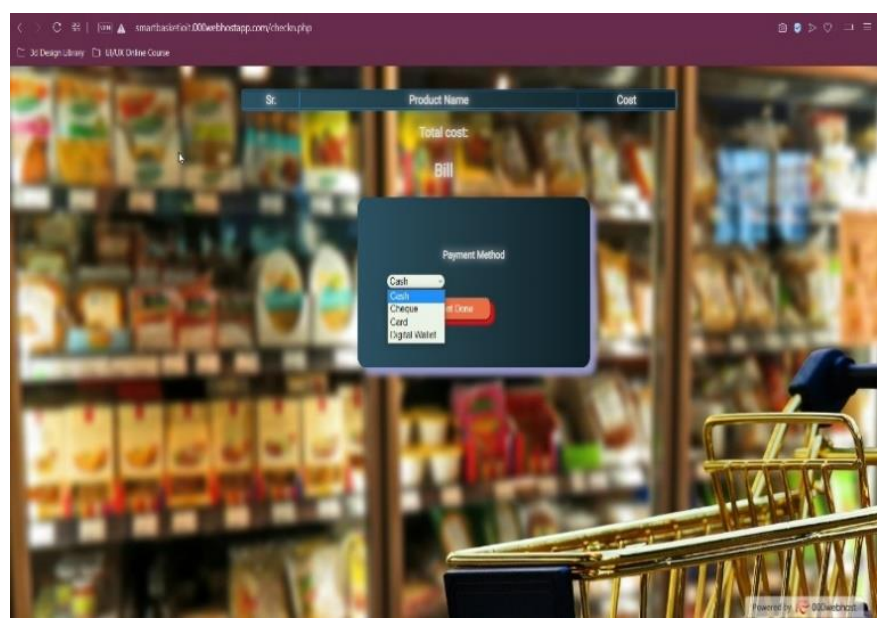

Figure 4: Check-in Page offering various payment modes for billing and invoice.

The image on left bottom is for the final check-out of the customer where he can choose the various mode of payment. The above image is for initial checking to ensure there is no fraud with him. When the customer is done with his shopping journey process, he can enter his basket ID (provided at the time when the customer enters the grocery store or mall) and come to this page for final billing.

\section{Database System}

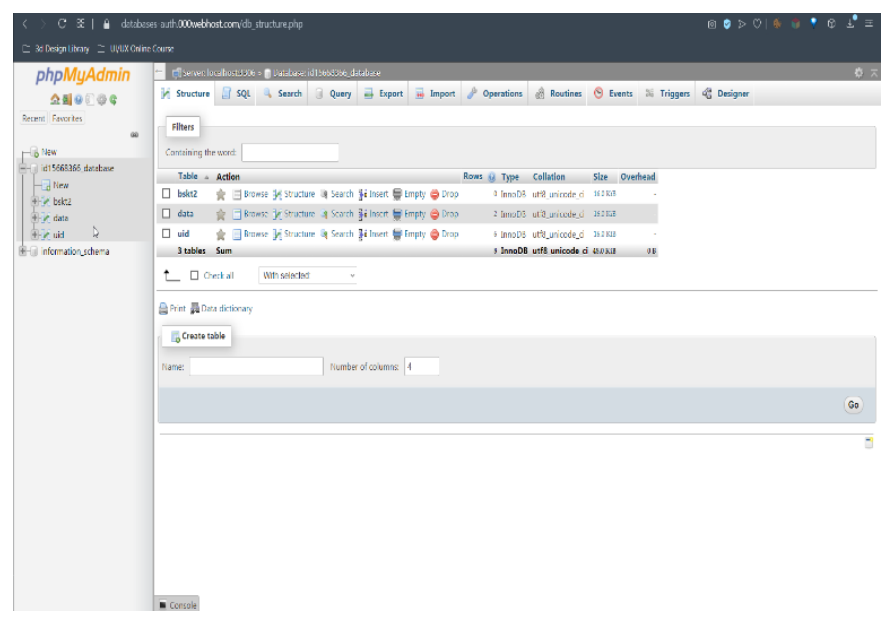

Figure 5: MySQL databases for Smart Basket

The above image shows the database used for Smart Basket. The admin used here is phpMyAdmin which uses MySQL database. The basket has three tables where data will store the product name and cost of the product. The user ID table will store user ID which is the customer's basket ID and data of the products which he scanned to store and display when requested for the get-fetch cycle process.

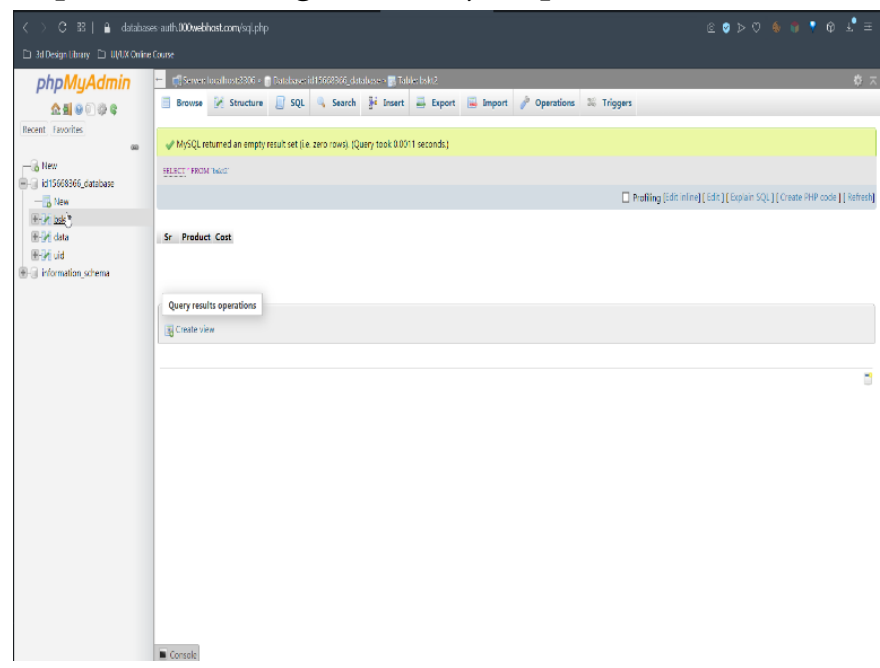

Figure 6: Basket table in MySQL (customer product table)

The first table is of the basket where the list of the products with cost will be stored and displayed when requested.

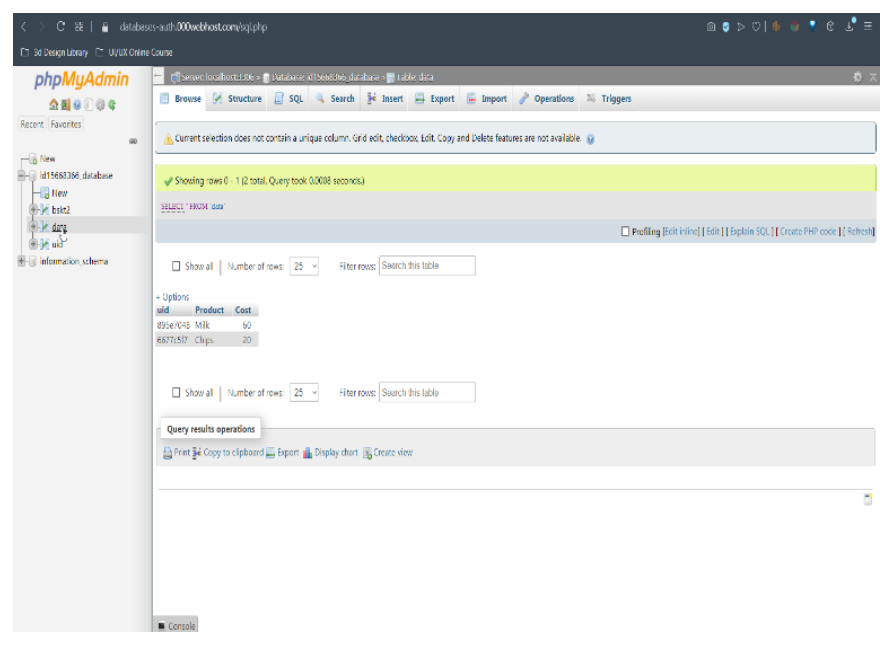

Figure 7: Product table having product name and cost stored in MySQL.

The second table is of data where the list of products is stored so when a customer scans a product the cost of the product and the product name will be fetched from this table. 


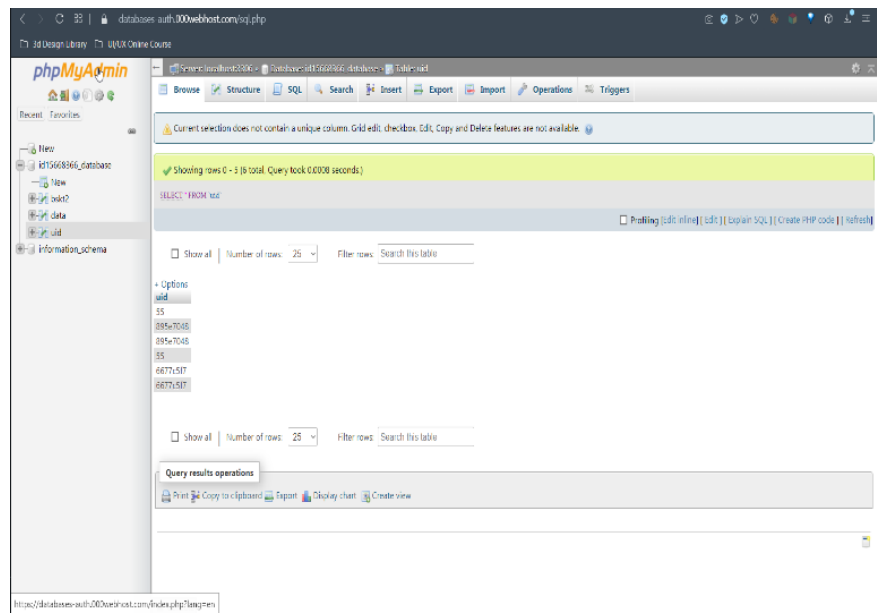

Figure 8: MySQL table of the customer (user ID table)

The third table consists of user ID which is assigned to the customer's basket for final checkout and in the process of the get-fetch-request cycle with the help of SPI and HTTP protocol to make the billing invoices fast and in an efficient manner.

\section{METHODOLOGY}

\section{RFID Technology}

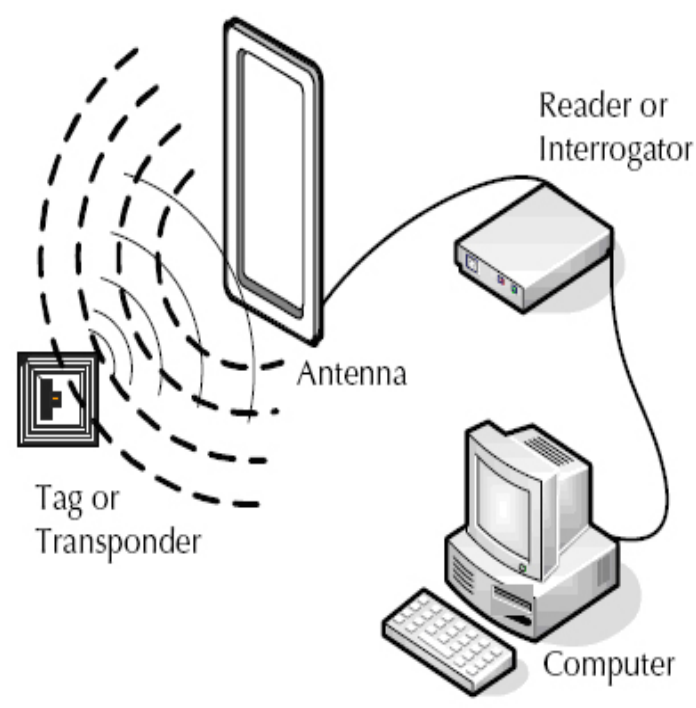

Figure 9: Basic Prototype of RFID Tag and Reader

RFID is a technology that stands for Radio Frequency Identification and is used to transfer data from devices in form of a wireless medium. Using this technology RFID tags allow users to automatically identify product barcode and details which help in identifying them with tracking of prices. [4]A good application of RFID technology is in IoT applications.

In this paper smart basket uses an RFID tag and RFID reader.

\section{RFID Tag}

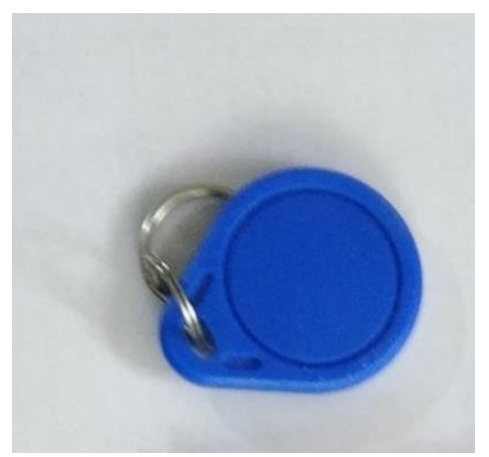

Figure 10: RFID Tag

These are used to track items using an RFID reader and antenna. The RFID tag, in a nutshell, comprises of two parts, first, is an antenna used basically for transmitting and receiving signals, and an RFID chip that tracks and stores the ID of the tag and other useful information.

RFID tags transmit signals with the help of radio waves to a reader or antenna or a combination of both. They work on radio waves generated by the reader, the energy work upon the internal antenna to tag's chip [4] [5]. The chip is activated by the energy which facilitates energy with useful information. This then transmits a signal back towards the antenna or reader.

\section{RFID Reader}

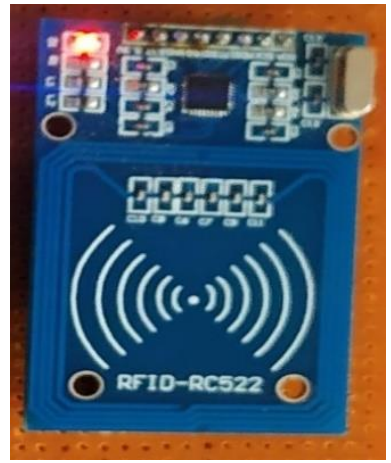

Figure 11: RFID Reader 
This is a main central part of the RFID system; it is the brain of the RFID system and is important for any system to function and work. The RFID reader is also called interrogators. The Reader transmits and receives radio waves to set a medium and communicate with RFID tags. There are two major types of RFID readers: Fixed RFID Readers and Mobile RFID Readers.

\section{Fixed RFID Readers:}

These RFID readers can be connected to eight different antennas using external antenna ports with one additional antenna that can be connected anywhere.

\section{Mobile RFID Readers:}

These RFID readers are easy to use since they allow flexibility by allowing them to work with mobile. So, they are handheld devices that can read RFID tags while doing other work. They can communicate with a smart device such as IoT Appliances or a host computer.

\section{Arduino}

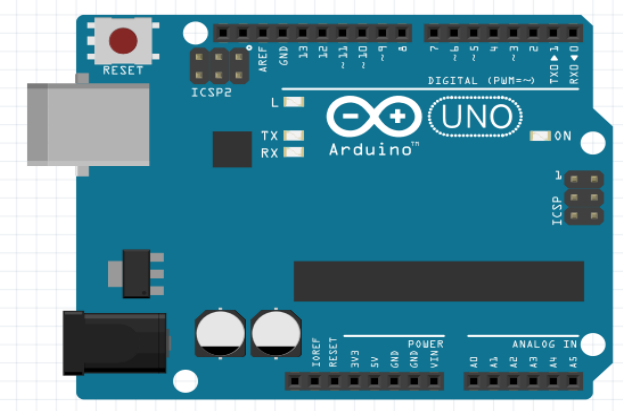

Figure 12: Arduino Hardware

Arduino is a hardware platform used for building electronic applications such as IoT, Digital circuits, LEDs, motors, etc. It is an open-source platform that consists of a piece of software or IDE that facilitates to program for hardware and another thing it consists of is a physical programmable circuit board such as a microcontroller [5]. The Arduino platform has made it easy to load code from software to hardware such as microcontroller without any changes. It simply loads the code which uses $\mathrm{C}++$ programming. It does not separate the hardware and software; it connects them with a USB cable. In this paper the smart basket stores the scanned product with RFID technology, and the memory is stored in a microcontroller that uses Arduino IDE to store and load the code for scanning the items. It processes data like CPU and helps to control all the components in the hardware.

\section{Node MCU ESP2866(Wi-Fi Module)}

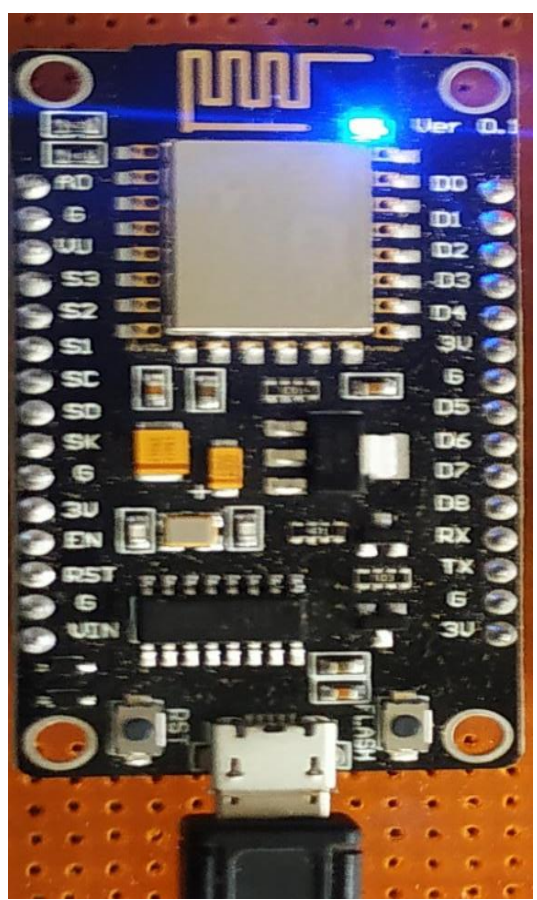

Figure 13: Node MCU ESP2866

Node MCU is an open-source LUA and developed for the Wi-Fi module. The Node MCU has a hardware design such that it makes it easy to edit/modify/build the module. Also, the ESP2866 is the most common chip used which is Wi-Fi enabled [6]. And it supports multiple protocols such as UART, SPI, I2C, etc. In this paper, the protocol used is SPI which is a serial protocol and can be connected to serial devices such as RFID tags and readers. 
ESP2866 is a Wi-Fi Module that helps to send and receive data and communicate with hardware components such as a microcontroller, RFID reader, etc. The ESP2866 uses the analogy same as used in with smartphones. The same process is followed by ESP2866 of sending data or transferring data from Wi-Fi to smartphones, In the similar way this module sends data from RFID reader to mobile phone and microcontroller. In this paper smart basket uses ESP2866 to store the data in our local database (MySQL) or store it in a cloud server to download and upload the details of the products scanned. This module facilitates to transfer of the details to the billing software from the basket.

\section{SCOPE OF IMPROVEMENT}

With the advent and popularity of IoT appliances, there exist security issues for the payment of customers during final check-in. So as cybersecurity is an improving field there will arise a need for improvement of payment gateway. The RDBMS is good for small businesses, but NoSQL databases can help grocery stores and enterprise-level businesses, to store data more efficiently without any concerns about data manipulation. The Smart Basket needs security enhancement and a efficient data processing and data management system which will help the product for betterment of the world and society in upcoming future.

\section{VII.CONCLUSION}

Considering the latest changes in IoT technology there are number of trends which are helping the world to grow a better place, so the Smart Basket IoT application will bring a change and make a new trend in retail shopping. This will become a necessity in the upcoming future for shopping and retail marketing industry, as it performs the fast billing. Further the cost of the Hardware module will decrease with new evolving technology, resulting in making more IoT embedded hardware. Smart Basket will help the marketing industry to build more hardware with less cost. The automation industry will upgrade the IoT products so this product will work seamlessly resulting less manpower.

\section{REFERENCES}

[1]. A. Kumar, A. Gupta, S. Balamurugan, S. Balaji, and R. Marimuthu, "Smart shopping cart," in 2017 International Conference on Microelectronic Devices, Circuits, and Systems (ICMDCS), Aug 2017, pp. 1-4. 4]

[2]. P. Aryan, "Smart shopping cart with automatic billing system through RFID and Bluetooth," International Journal of Emerging Technology and Computer Science, vol. 1, no. 2, 2016.

[3]. P. Chandrasekar and T. Sangeetha, "Smart shopping cart with automatic billing system through RFID and Zigbee," 2014 International Conference on Information Communication and Embedded Systems, ICICLES 2014, 02 2015.

[4]. Kalyani Dawkhar, Shraddha Dhomase, Samruddhi Mahabaleshwarkar "Electronic Shopping Cart for Effective Shopping based on RFID” International Journal of Innovative Research in Electrical, Electronic, Instrumentation and Control Engineering Vol. 3, Issue 1 pp 84-86, January 2015.

[5]. Galande Jayashree, Rutuja Gholap, Priti Yadav on "RFID based Automatic billing trolley" year - 2015, publication - IJETAE.

[6]. D. Mohanapriya, R. Mohamed 6]Anas, P. Nandhini, N.M Deepika, "Design and implementation of Smart Basket Cart Using Near Field Communication", Indian Journal of Emerging Electronics in Computer Communications Vol.5, Issue 1-2018, ISSN: 2393-8366 
[7]. K. Lalitha, M. Ismail, Sasi Kumar Gurumurthy,

A. Tejaswi, "Design of an Intelligent Shopping

Basket using IoT", 2017 International Journal of

Pure and Applied Mathematics 151(1):1311-

8080.

[8]. V. Sharma, S. Malhotra, and M. Hashmi, "An

Emerging Application Centric RFID

Framework Based on New Web Technology,"

2018 IEEE International Conference on RFID

Technology \& Application (RFID-TA), Macau, 2018, pp. 1-6. doi: 10.1109/RFIDTA.2018.8552742.

[9]. Y.A. Badamasi, "The working Principle of an Arduino", in Electronics, computer, and computation (icecco), 11th International Conference on INFORMATION COMMUNICATION EMBEDDED SYSTEMS, pages 1-4, 2014.

[10]. Jadhav Rahul, Pradeep, Nandkumar, Tarali Shivkumar "RFID based Automated billing trolley", year -2015, publication - IJSRD.

[11]. Rachana Doshi, Amrita Sutar, Sonali Aher, Sanvida Dalvi "RFID Based Smart Trolley for Automatic Billing System”, year -2017, publication- IJESC.

\section{Cite this article as :}

Talreja Sahil, Pendharkar Arjun, Madur Srushti, Mohammad Saad Nalband, Pragati Mahale, "Smart Basket : The Modern era IoT Application", International Journal of Scientific Research in Science and Technology (IJSRST), Online ISSN : 2395-602X, Print ISSN : 2395-6011, Volume 8 Issue 2, pp. 585-593, March-April 2021. Available at doi : https://doi.org/10.32628/IJSRST218273

Journal URL : https://ijsrst.com/IJSRST218273 